\title{
CONJUGATE PURITY AND INFINITE GROUPS
}

\author{
BOLA O. BALOGUN
}

(Received 28 February; revised 31 October 1978)

Communicated by H. Lausch

\begin{abstract}
In Balogun (1974), we proved that a finite group in which every subgroup is conjugately pure is necessarily Abelian and we left open the infinite case. In this paper we settle this problem positively for soluble, locally soluble groups and certain classes of groups which include the $F C$-groups. In the last section of this paper we characterize groups which are conjugately pure in every containing group.
\end{abstract}

Subject classification (Amer. Math. Soc. (MOS) 1970): 20 E 99.

\section{Introduction}

A subgroup $H$ of a group $G$ is said to be conjugately pure in $G$ if whenever two elements of $H$ are conjugate in $G$ they are already conjugate in $H$. This concept plays a fundamental role in the theory of groups. For example, a classical result of Burnside asserts that if a finite group $G$ contains a conjugately pure Sylow $p$ subgroup, then $G$ has a normal $p$-complement. For this result and its various generalizations, see Huppert (1967), p. 432; Passman (1968), p. 103; and Friesen (1974).

We shall use the following notations. Unexplained notations will be as in Robinson (1972).

$H P$ : class of all groups in which every subgroup is conjugately pure

$\mathfrak{F}$ : class of all finite groups

S: class of all soluble groups

$L S$ : class of all locally soluble groups

$\mathfrak{A}$ : class of all Abelian groups 
In this paper we are mostly concerned with the following problem (Balogun (1974)):

For which classes $\mathfrak{X}$ of groups is

$$
\mathfrak{X} \cap(H P) \leqslant \mathfrak{A} \text { valid? }
$$

In Balogun (1974), we proved that (1.1) holds if $\mathfrak{X}=\mathfrak{F}$. In this paper we shall prove that (1.1) is valid for $\mathfrak{X}=\mathfrak{S}, L \subseteq$ or certain classes of groups which include the $F C$-groups.

\section{The property $\mathfrak{P}$ and finite groups}

Our investigation of groups with property $H P$ lead naturally into the notion of $\mathfrak{P}$-groups which we now define.

A group $G$ is said to have property $\mathfrak{P}$ if $H^{\prime}=H^{\prime \prime}$ for every subgroup $H$ of $G$.

The class of $\mathfrak{B}$-groups contains the class of all Abelian groups. Whether there are such groups other than the Abelian groups is an unsolved problem. However, the following example of Baumslag (1969) is quite close to the type of group we are looking for.

EXAMPLE. Let $G=\left\langle a, b \mid a=\left[a, a^{b}\right]\right\rangle$; then every subgroup $H$ of finite index satisfies $H^{\prime} \neq H^{\prime \prime}$. However, if $H=\left\langle a, a^{b} \mid a=\left[a, a^{b}\right]\right\rangle$ then $H^{\prime \prime}=H^{\prime}$.

LEMMA 1.1. Let $G$ be any group which has the property HP or $\mathfrak{P}$ and suppose that the derived group $G^{\prime}$ of $G$ is Abelian. Then $G$ itself is Abelian.

Proof. If $G$ has property $\mathfrak{P}$, then $G^{\prime}=G^{\prime \prime}=1$ and so $G$ is Abelian.

In an $H P$-group every normal Abelian subgroup is central. Now assume that $G$ is not Abelian, say $x y \neq y x$ so that $x^{\nu}=x[x, y] \neq x$. Let $H=\langle x,[x, y]\rangle$. Then $H$ is Abelian, but $x^{y} \neq x$ so that $H$ is not conjugately pure, a contradiction.

THEOREM 1.1. Every HP-group is a $\mathfrak{B}$-group.

Proof. Let $H$ be a subgroup of an $H P$-group $G$. By Balogun (1974), Lemma 1, every section of an $H P$-group is an $H P$-group and hence $H / H^{\prime \prime}$ is an $H P$-group Furthermore, $\left(H / H^{\prime \prime}\right)^{\prime} \cong H^{\prime} / H^{\prime \prime}$ is Abelian; hence $H^{\prime}=H^{\prime \prime}$ by Lemma 1.1.

As another consequence of Lemma 1.1 we now have

THEOREM 1.2. Every soluble $\mathfrak{P}$-group is Abelian.

LEMMA 1.2. If $G$ is a $\mathfrak{P}$-group, then so is every section of $G$. 
Proof. Let $H / N$ be a section of $G$. Then $H^{\prime \prime}=H^{\prime}$. Hence

$$
(H / N)^{n} \cong H^{\prime \prime} N / N=H^{\prime} N / N \cong(H / N)^{\prime} .
$$

The Theorem of Balogun (1974) can be deduced from

THEOREM 1.3. A finite $\mathfrak{P}$-group is Abelian.

ProOF. Let $G$ be a counterexample of minimal order. We may assume that $G$ is not a $p$-group. If $p$ is a prime which divides $G$, let $H$ be a Sylow $p$-subgroup of $G$. Consider its normalizer $N_{G}(H)$. If $N_{G}(H)=G$ then $H$ is a proper normal subgroup of $G$. Hence $G / H$ and $H$ are both of orders less than the order of $G$ and so they are both Abelian. Hence $G$ is soluble, and so $G$ is Abelian-a contradiction.

If $N_{G}(H)<G, N_{G}(H)$ is Abelian and $H \leqslant N_{G}(H)=C_{G}(H)$. Hence by Burnside's Theorem (Passman (1968), p. 103), $H$ has a normal p-complement in $G$. Thus as before this implies that $G$ is Abelian, contrary to supposition.

\section{The property $\mathfrak{P}$ and generalized soluble groups}

We have already seen that every soluble $\mathfrak{P}$-group is Abelian. So it is natural to ask if this result can be extended to any classes of generalized soluble groups. The widest known class of generalized soluble groups is the class $S N$. More precisely we want to know if the diagram of classes of groups in Robinson (1972), Part 2, p. 80 collapses when each vertex is intersected with $\mathfrak{P}$.

THEOREM 2.1. A locally soluble $\mathfrak{P}-$ group is Abelian.

Proof. Let $G \in(L S) \cap \mathfrak{P}$. Let $x, y \in G$. Then since $G \in L S$, there exists a group $H \in \Im$ so that $x, y \in H$. Now $\langle x, y\rangle$, as a subgroup of $G$, is in $\mathfrak{P}$ since $\mathfrak{P}$ is $\subseteq$-closed. Also $\langle x, y\rangle$, as a subgroup of $H$, is in $\mathfrak{S}$. Hence $\langle x, y\rangle \in \mathfrak{S} \cap \mathfrak{P}=\mathfrak{A}$.

THEOREM 2.2. Let $G$ be an FC-group with property $\mathfrak{P}$. Then $G$ is Abelian.

Proof. By Robinson (1972), Theorem 4.32, $G^{\prime}$ is finite and hence Abelian (by Theorem 1.3). So by Lemma 1.1, $G$ is Abelian.

THEOREM 2.3. Let $G$ be an FC-hypercentral group with property $\mathfrak{P}$, then $G$ is Abelian.

Proof. Let $\left\{F_{\alpha}\right\}$ be the upper $F C$-central series of $G$. We shall prove by transfinite induction that each $F_{\alpha}$ is Abelian. Now $F_{0}=1$ is Abelian. So we assume that we 
already know $F_{\alpha}$ is Abelian for all $\alpha<\beta$. Then if $\beta-1$ exists, we have that $F_{\beta-1}$ is Abelian. Now $F_{\beta} / F_{\beta-1}$ is an $F C$-group with property $\mathfrak{P}$, hence it is Abelian. Thus $F_{\beta}$ is a soluble $\mathfrak{P}$-group and so $F_{\beta}$ is Abelian. On the other hand, if $\beta$ is a limit ordinal, we have $F_{\beta}=\bigcup_{\alpha<\beta} F_{\alpha}$ is the ascending union of Abelian groups and so it is Abelian.

QUESTION. If $A$ and $B$ are $\mathfrak{P}$-groups, is their direct product $A \times B$ a $\mathfrak{P}$-group?

\section{Groups which are conjugately pure in every group in which they are contained as subgroups}

THEOREM 3.1. A group $H$ is conjugately pure in every group $G$ in which it is embedded if and only if all elements of the same order in $H$ are conjugates of each other.

Proof. The condition is clearly sufficient, since any two elements of $H$ which are images under an automorphism of $G$ have the same order. On the other hand, by a result of Higman, Neumann and Neumann (1949), every group $H$ can be embedded in a group $G_{0}$ in which all elements of the same order are conjugate. Thus, if $H$ does not have this property, then $H$ is not conjugately pure in $G_{0}$.

Remark. In fact groups $H$ which satisfy the condition of Theorem 3.1 are characteristically pure in every group $G$ with $H \leqslant G$, in the sense that any two elements of $H$ which are images of each other under an automorphism of $G$ are conjugates in $H$.

THEOREM 3.2. A group $H$ is conjugately pure in every group $G$ in which it is a normal subgroup if and only if $H$ has the (locally inner) property that any two elements which are images under an automorphism of $H$ are conjugate in $H$.

Proof. If we define an extension of $H=\{(1, h) \mid h \in H\}$ by $A=\operatorname{Aut}(H)$ as the group

$$
B=\{(a, h) \mid h \in H, a \in A\}
$$

with $\left(a_{1}, h_{1}\right)\left(a_{2}, h_{2}\right)=\left(a_{1}, a_{2}, h_{1}^{a_{2}} h_{2}\right)$, that is, $B=\operatorname{Hol}(H)$, the holomorph of $H$, then the locally inner condition of the theorem is equivalent to the assertion that $H$ is conjugately pure in its holomorph.

If $H$ is conjugately pure in its holomorph then it is, in fact, conjugately pure in every group $G$ with $H \triangleleft G$. 
QUESTION. Is it true that if all subgroups of a group $G$ are characteristically pure then $|G|=1$ or 2 ?

We could define characteristic purity in the following weak sense: Let $H \leqslant G$, $H$ is said to be weakly characteristically pure in $G$ if whenever an automorphism of $G$ maps $h \rightarrow h^{\prime}\left(h, h^{\prime} \in H\right)$ then there is an automorphism of $H$ which maps $h \rightarrow h^{\prime}$.

For example, every characteristic subgroup of a group is weakly characteristically pure. Thus in a cyclic group every subgroup is weakly characteristically pure. Also there are many non-Abelian finite groups in which every subgroup is weakly characteristically pure, for example the quarternion group.

Problem. Characterize all finite groups in which every subgroup is weakly characteristically pure.

For a finite Abelian p-group $G$, we can write $G=C_{e_{1}} \times C_{e_{2}} \times \ldots \times C_{e_{k}}$ with $e_{k}|\ldots| e_{2} \mid e_{1}$, and if $G$ is weakly characteristically pure, we must have $k=1$ or $e_{2}=\ldots=e_{k}=p$. To prove this we note first of all that two elements of $G$ can be mapped into each other by an automorphism of $G$ if and only if they have the same orders and the same divisibilities. Thus if $C_{e_{i}}=\left\langle x_{i}\right\rangle(i=1,2, \ldots, k)$, then $x_{i}$ has order $e_{i}$ and divisibility by all integers relatively prime to $e_{i}$. For any fixed $i \neq 1$, the two elements $x_{i}$ and $\left(e_{1} / e_{i}\right) x_{1}+x_{i}$ have equal orders and divisibilities. Hence there is an automorphism $\alpha$ of $G$ with $x_{1}^{\alpha}=\left(e_{1} / e_{i}\right) x_{1}+x_{i}$. If $e_{i} \neq p(i \neq 1)$, then the subgroup $H=\left\langle p x_{i},\left(e_{1} / e_{i}\right) x_{1}+x_{i}\right\rangle$ is not weakly characteristically pure in $G$ since

$$
\left(p x_{i}\right)^{\alpha}=\left(p e_{1} / e_{i}\right) x_{1}+p x_{i},
$$

where $y=\left(p e_{1} / e_{i}\right) x_{1}+p x_{i}$, but not $p x_{i}$, is divisible by $p$ in $H$. Therefore no automorphism of $H$ maps $p x_{i}$ to $y$, contradicting the fact that $G$ is weakly characteristically pure. Hence $e_{2}=\ldots=e_{k}=p$.

\section{Acknowledgement}

The author wishes to thank the referee for advice during the writing of this paper.

\section{References}

B. O. Balogun (1974), 'Conjugately pure subgroup problems', Amer. Math. Monthly 81, 156-158. (MR 48 \# 11332.)

G. Baumslag (1969), 'A non-cyclic one-relator group all of whose finite quotients are cyclic', J. Austral. Math. Soc. 10, 497-498. 
D. K. Friesen (1974), 'Normal complements in finite solvable groups', J. Austral. Math. Soc. 18, 262-264. (MR 51 \# 51749.)

G. Higman, B. H. Neumann and Hanna Neumann (1949), 'Embedding theorems for groups', J. London Math. Soc. 24, 247-254.

B. Huppert (1967), Endliche Gruppen I (Die Grundlehren der Mathematischen Wissenschaften, Band 134, Springer-Verlag, Berlin, New York).

D. S. Passman (1968), Permutation groups (W. A. Benjamin Inc., New York, Amsterdam).

D. J. S. Robinson (1972), Finiteness conditions and generalized soluble groups, Parts 1 and 2 (Ergebnisse der Mathematik und ihrer Grenzgebiete, Springer-Verlag, Berlin, Heidelberg, New York).

University of Ife

Ile-Ife

Nigeria 